\title{
A cluster of tertiary transmissions of 2019 novel coronavirus (SARS-CoV-2) in the community from infectors with common cold symptoms
}

Nam Joong Kim 1,, , Pyoeng Gyun Choe ${ }^{1, *}$, Sang-Joon Park², Jaegyun Lim³, Wang Jun Lee ${ }^{4}$, Chang Kyung Kang ${ }^{1}$, Wan Beom Park ${ }^{1}$, Moon-Woo Seong ${ }^{5}$, and Myoung-don Oh ${ }^{1}$

\begin{abstract}
${ }^{1}$ Department of Internal Medicine, Seoul National University College of Medicine, Seoul; Departments of ${ }^{2}$ Internal Medicine, ${ }^{3}$ Laboratory Medicine, and ${ }^{4}$ Surgery, Myongji Hospital, Goyang; ${ }^{5}$ Department of Laboratory Medicine, Seoul National University College of Medicine, Seoul, Korea
\end{abstract}

Received: March 31, 2020

Revised : May 7, 2020

Accepted: May 7, 2020

\section{Correspondence to}

Myoung-don Oh, M.D.

Department of Internal

Medicine, Seoul National

University College of Medicine,

103 Daehak-ro, Jongno-gu, Seoul, 0308o, Korea

Tel: +82-2-2072-2945

Fax: +82-2-762-9662

E-mail: mdohmd@snu.ac.kr https://orcid.org/0000-00022344-7695

*These authors contributed equally to this work.
Background/Aims: As the global impact of the novel coronavirus disease 2019 (COVID-19) has been severe, many countries have intensified containment activities to eliminate virus transmission, through early detection and isolation strategies. To establish a proper quarantine strategy, it is essential to understand how easily the virus can spread in the communities.

Methods: In this study, we collected detailed information on the circumstances in which human-to-human transmission occurred in the tertiary transmission cases of COVID-19 in the community.

Results: On January 26, 2020, an imported case of COVID-19 was confirmed, and by February 10, 2020, one secondary transmission and three tertiary transmissions were identified. Secondary transmission occurred on the first day of illness of the infector, and his symptoms were suggestive of a common cold. The transmission occurred during a 9o-minute long meal together in a restaurant. The people were sitting within one meter of each other, and had no direct contact. The tertiary transmission also occurred on the first-day illness of the other infector, and his only symptom was slight chills. The transmission occurred at a church during 2-hour-long worship, and two rows separated them.

Conclusions: Our findings suggest that mildly symptomatic patients with COVID-19 could transmit the virus from the first day of illness through daily activities in the community. Early detection and isolation of patients with COVID-19 may be challenging.

Keywords: Coronavirus; Severe acute respiratory syndrome coronavirus 2; COVID-19; Common cold; Transmission

\section{INTRODUCTION}

In December 2019, a new strain of betacoronavirus, the severe acute respiratory syndrome coronavirus 2 (SARS-CoV-2), emerged in Wuhan, Hubei Province, China, causing a significant outbreak of pneumonia [1]. The number of cases of novel coronavirus disease 2019
(COVID-19) has increased rapidly in Hubei Province and the major cities in China, such as Guandong, Zhejiang, and Henan [2]. Subsequently, the epidemic spread quickly worldwide and World Health Organization (WHO) on March 11, 2020 declared COVID-19 a pandemic. As of March 29, 2020, 148 countries have reported local transmission of COVID-19 [3]. 
Some countries, such as Korea, have been trying to contain the COVID-19 outbreak to eliminate the virus from nations. This strategy of containment for elimination primarily relies on early detection and isolation of cases. Assuming that the basic reproduction number $\left(\mathrm{R}_{\mathrm{o}}\right)$ of SARS-CoV-2 is 2.2 [4], more than half of the cases have to be identified and isolated before the infectors transmit the virus to other persons. However, transmission of the virus by infectors during the period of symptom development (prodromal phase), or by those with mild symptoms, could make early detection and isolation of infectors more challenging.

Here, we report a cluster of secondary and tertiary transmissions of SARS-CoV-2 in a community in South Korea. The secondary transmission occurred on the first day of illness of the infector, while his symptoms were mild and suggestive of a common cold. The transmission occurred at a restaurant. The tertiary transmission also happened on the first day of illness of the other infector, and his only symptom was slight chills. The transmission occurred during worshiping in the church.

\section{METHODS}

\section{Cases}

This study included one imported case of SARS-CoV-2 infection from Wuhan, China [5], one secondary transmitted case, and three tertiary transmitted cases, which was confirmed between January 26 and February 5, 2020 in South Korea. Laboratory diagnostic confirmation of SARS-CoV-2 infection was performed by the Korea Centers for Disease Control and Prevention (KCDC) using real-time reverse transcriptase-polymerase chain reaction (rRT-PCR) according to the modified WHO proto$\operatorname{col}[6]$.

\section{Data collection}

We recorded and analyzed epidemiological, demographical, and clinical findings using a modified standardized case record form of the WHO early investigation protocol [7]. The board-certified infectious disease specialists conducted an in-depth interview with each patient to obtain detailed data relating to human-to-human transmission. Clinical outcomes were followed-up on February 29, 2020. During hospitalization, rRT-PCR was conducted on respiratory specimens by the laboratories in each hospital using the Kogene PowerChekTM 2019 novel coronavirus real-time-PCR kit (Kogenebiotech, Seoul, Korea) according to the manufacturer's recommendations.

This study was approved by the Institutional Review Board of the Seoul National University Hospital (number 2002-008-1096) and Myongji Hospital (number 2020-01-017). Written consent was obtained from the patients.

\section{RESULTS}

From January 25 to February 5, 2020, five patients with COVID-19 were admitted to the study hospitals (Fig. 1). Of the five patients, one was an imported case from $\mathrm{Wu}-$ han, China, one was a secondary transmitted case, and three were tertiary transmitted cases (Table 1).

On January 20, 2020, a 54-year-old man (index patient) reached Korea from Wuhan, China. He had no history of animal contact or market visits, including the Huanan seafood wholesale market in Wuhan. On January 22, 2020, he developed fever, myalgia, and took antipyretics. As he felt better after medication intake, he went out to meet his friend (patient 1). On January 25, 2020, he contacted the public health center due to worsening fever and was confirmed with COVID-19 by KCDC on January 26. On admission (5th day of illness), the index patient denied any respiratory symptoms, and the chest radiograph was normal (Fig. 2A). On January 30, 2020 (9th day of illness), he began to complain of productive cough and shortness of breath, and the high resolution computed tomography (HRCT) of the lung showed bilateral ground-glass infiltrates (Fig. 2B).

Patient 1 was a 55-year-old man with a medical history of diabetes mellitus and early gastric cancer. On January 22, 2020, he had a meal with the index patient at a restaurant. Patient 1 failed to notice any symptoms in the index patient, such as coughing or sneezing during their meal together, and also had no direct contact with him, including shaking hands, kissing on the cheek, or hugging. The distance between the index patient and patient 1 was less than $1 \mathrm{~m}$, and the duration of their meeting was approximately 90 minutes. On January 26, 2020, the patient 1 developed mild chills but showed no 


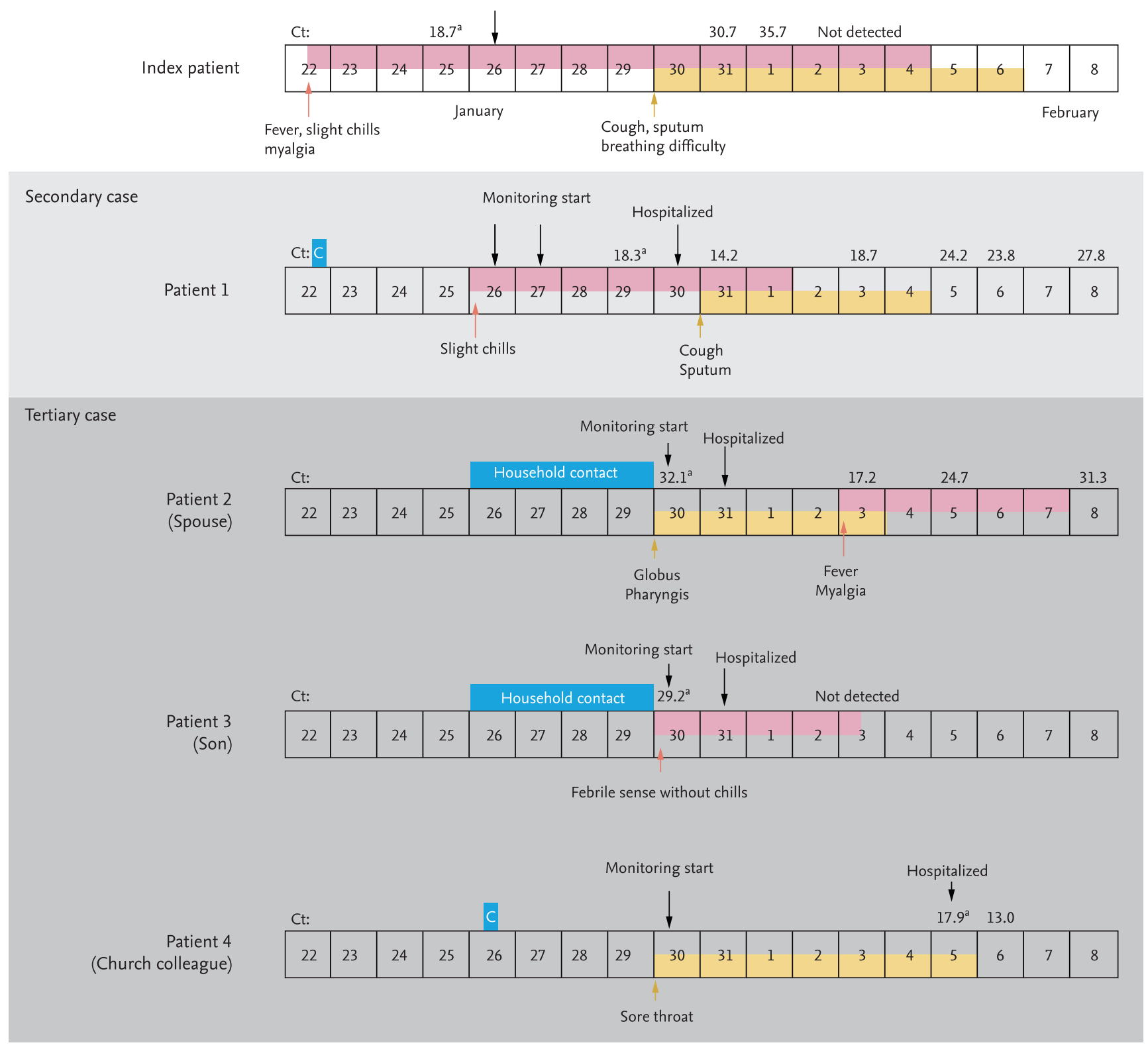

Constitutional symptoms

Respiratory symptoms

Figure 1. Timeline of exposure and clinical course of the patients from January 22, 2020 to February 8, 2020. Cyclic threshold (Ct) values from real-time reverse transcriptase-polymerase chain reaction to test for severe acute respiratory syndrome coronavirus 2 (SARS-CoV-2) E gene. ${ }^{a}$ The results marked are the results conducted by the Korea Centers for Disease Control and Prevention using the modified World Health Organization protocol, and the rest are the results conducted by the laboratory in each hospital using the Kogene PowerChek ${ }^{\mathrm{TM}}$ 2019-nCoV Real-time-PCR kit (Kogenebiotech).

serious illness and attended the church service as usual. On January 27, 2020, KCDC informed the patient 1 that the index patient was confirmed to have SARS-CoV-2 infection, and hence, he adopted self-quarantine measure. Patient 1 was not classified as a close contact of the index patient in the initial epidemiological investigation as the timing of the onset of symptoms in the index patient was assumed to be after meeting patient 1. However, on January 29, 2020, this timing was revealed to be before meeting patient 1 , and KCDC reclassified 
Table 1. Summary of epidemiological and clinical features of the patients

\begin{tabular}{|c|c|c|c|c|c|}
\hline Variable & Index patient & Patientı & Patient2 & Patient3 & Patient4 \\
\hline $\begin{array}{l}\text { Transmission } \\
\text { generation in Korea }\end{array}$ & Primary & Secondary & Tertiary & Tertiary & Tertiary \\
\hline Relation & & $\begin{array}{l}\text { Friend of index } \\
\text { patient }\end{array}$ & Spouse of patient 1 & Son of patient 1 & $\begin{array}{l}\text { Church colleague } \\
\text { of patient } 1\end{array}$ \\
\hline Age/Sex & 54/Male & 55/Male & 54/Female & 24/Male & 59/Female \\
\hline Occupation & Office worker & Office worker & Housewife & Student & Housewife \\
\hline Underlying conditions & None & $\begin{array}{l}\text { Diabetes mellitus } \\
\text { Early gastric cancer }\end{array}$ & $\begin{array}{l}\text { Hashimoto } \\
\text { thyroiditis }\end{array}$ & None & Diabetes mellitus \\
\hline Date of exposure & $-20 \mathrm{Jan}$ & $22 \mathrm{Jan}$ & 26 Jan-29 Jan & 22 Jan-29 Jan & 26 Jan \\
\hline Place of exposure & Wuhan, China & Restaurant & Household & Household & Church \\
\hline Duration of exposure & & $2 \mathrm{hr}$ & 4 day & 4 day & $2 \mathrm{hr}$ \\
\hline $\begin{array}{l}\text { Self-quarantine \& } \\
\text { monitoring started on }\end{array}$ & & 27 Jan & $30 \mathrm{Jan}$ & $30 \mathrm{Jan}$ & $30 \mathrm{Jan}$ \\
\hline Date of first symptom & 22 Jan & 26 Jan & 30 Jan & 30 Jan & 30 Jan \\
\hline First symptom & $\begin{array}{l}\text { Febrile sense, } \\
\text { myalgia }\end{array}$ & Chilly sense & Globus pharyngis & Febrile sense & Sore throat \\
\hline $\begin{array}{l}\text { Max temperature } \\
\text { during active } \\
\text { monitoring }\end{array}$ & & Not checked & Not checked & $37.8^{\circ} \mathrm{C}$ & $36.7^{\circ} \mathrm{C}$ \\
\hline \multicolumn{6}{|l|}{ rRT-PCR results } \\
\hline First Ct value (date) & 18.7 (26 Jan) & 18.3 (30 Jan) & 32.1 (31 Jan) & 29.2 (31 Jan) & $17.9(5 \mathrm{Feb})$ \\
\hline Lowest Ct value (date) & 18.7 (26 Jan) & $14.2(31 \mathrm{Jan})$ & $17.2(3 \mathrm{Feb})$ & 29.2 (31 Jan) & $13.0(6 \mathrm{Feb})$ \\
\hline \multicolumn{6}{|l|}{ Radiologic findings } \\
\hline First CXR (date) & $\begin{array}{l}\text { No active lung } \\
\text { lesion ( } 26 \text { Jan) }\end{array}$ & $\begin{array}{l}\text { No active lung } \\
\text { lesion (30 Jan) }\end{array}$ & $\begin{array}{l}\text { No active lung } \\
\text { lesion (31 Jan) }\end{array}$ & $\begin{array}{l}\text { Suspicious small } \\
\text { patch consolida- } \\
\text { tion ( } 31 \mathrm{Jan})\end{array}$ & $\begin{array}{l}\text { No active lung } \\
\text { lesion }(6 \mathrm{Feb})\end{array}$ \\
\hline First HRCT (date) & $\begin{array}{l}\text { Multiple patch } \\
\text { GGOs (30 Jan) }\end{array}$ & $\begin{array}{l}\text { Multiple patchy or } \\
\text { nodular } \\
\text { infiltration (31 Jan) }\end{array}$ & $\begin{array}{l}\text { A faint small GGO } \\
\text { (31 Jan) }\end{array}$ & $\begin{array}{l}\text { Subpleural } \\
\text { nodular lesion } \\
\text { (31 Jan) }\end{array}$ & Focal GGO (7 Feb) \\
\hline $\begin{array}{l}\text { Max } \mathrm{O}_{2} \text { requirement, } \\
\text { the 1st wk of illness }\end{array}$ & None & None & None & None & None \\
\hline $\begin{array}{l}\text { Max } \mathrm{O}_{2} \text { requirement, } \\
\text { the } 2 \text { nd wk of illness }\end{array}$ & $4 \mathrm{~L} / \mathrm{min}$ & None & None & None & None \\
\hline Clinical course & Stable & Stable & Stable & Stable & Stable \\
\hline
\end{tabular}

rRT-PCR, real-time reverse transcriptase-polymerase chain reaction; Ct, cyclic threshold; CXR, chest radiography; HRCT, high resolution computed tomography of the lung; GGO, ground-glass opacity.

patient 1 as close contact of the index patient. At the time of reclassification, patient 1 reported improvement of his condition, but KCDC decided to conduct a surveillance test for COVID-19. On January 30, 2020, he was diagnosed with SARS-CoV-2 infection and was admitted to the isolation unit. On admission, he had a mild fever (body temperature $37.9^{\circ} \mathrm{C}$ ) but denied any respiratory symptoms. The initial chest radiography was also normal (Fig. 2C). On the next day (6th day of illness), patient 1 began to complain of productive cough, and HRCT of the lung showed bilateral focal ground-glass opacities (Fig. 2D). He received supportive care, and neither an antiviral drug nor interferon was prescribed. His body temperature gradually decreased and returned to nor- 



Figure 2. Radiological findings from the patients. (A, C) Initial chest radiograph of the infectors (index patient and patient 1 , respectively) taken on admission showing normal lung. (B, D) High resolution computed tomography images obtained from index patient on 9 th day of illness and from patient 1 on 6 th day of illness, respectively, showing multiple patchy or nodular infiltration with peribronchovascular distribution in both lung fields.

mal on February 2, 2020 (8th day of illness).

Patient 2 and 3 were the spouse and son of the patient 1, respectively, and living with him in the same house. During the self-quarantine period of patient 1, they used separated spaces, distinct toilets, and usually wore a facial mask. On January 30, 2020, when patient 1 was diagnosed with COVID-19, KCDC collected respiratory samples from patients 2 and 3 for surveillance testing, although no symptoms were recorded. On January 31, 2020 , patients 2 and 3 were confirmed to have SARSCoV-2 infection and were admitted to the isolation unit. On admission, the symptoms of patients 2 and 3 were so 
minor that they barely had any discomfort during the in-depth interview. Patient 2 had experienced globus pharyngis one day before admission and had no fever on the day of admission. Patient 3 had felt slightly feverish without chills and myalgia one day before admission. On February 3, 2020 (9th day in the hospital), patient 2 developed fever and myalgia, which lasted about 5 days, and then showed signs of improvement.

Patient 4 was a 59-year-old woman with a medical history of diabetes mellitus and hyperlipidemia. On January 26, 2020, patient 4 attended 2-hour-long worship in a church with patient 1 . She sat two rows ahead of patient 1, and had no direct contact with him. On January 30, 2020, patient 4 developed a mild sore throat. On February 5,2020 , patient 4 was diagnosed with SARS-CoV-2 infection and was isolated; however, she had no fever and denied any symptoms other than a sore throat.

As of February 29, 2020, all the patients have been removed from the isolation room after two consecutive respiratory specimens collected at 48 -hour intervals tested negative for SARS-CoV-2 by rRT-PCR assay.

\section{DISCUSSION}

Our study demonstrates that secondary and tertiary transmission of SARS-CoV-2 occurs in the community. In both secondary (index patient $\rightarrow$ patient 1 ) and tertiary (patient $1 \rightarrow$ patient 4 ) transmission, the duration and distance of exposures were less than 2 hours and $1 \mathrm{~m}$, respectively. The transmissions occurred during everyday life activities, such as while having meals together with friends and going to church for worshiping.

It should be noted that SARS-CoV-2 was transmitted by infectors with mild, nonspecific symptoms, similar to the common cold. For instance, the index patient showed no respiratory symptoms, such as cough, sneezing, rhinorrhea, or productive sputum, while meeting his friend (patient 1 ), but still able to transmit the virus to patient 1. Patient 1, who was devoid of any respiratory symptoms, could also transmit the virus to a church colleague (patient 4). In addition, both index patient and patient 1 transmitted the virus on the 1st day of illness. As the infectors only show symptoms of common cold, it can be challenging to detect the infectors of COVID-19 without laboratory tests.
Our study also highlights that the transmission of SARS-CoV-2 may occur in the community during everyday life activities. Although the infectors (index patient and patient 1) had symptoms on the day of transmission, their symptoms were very mild to seek medical attention and hence, continued with their usual activities. Patients 2, 3, and 4 also had mild symptoms during the prodromal phase of their illness. Furthermore, without active monitoring and home quarantine, these patient would also have carried on with their daily routine, thus, transmitting the virus to other persons. Owing to their mild and nonspecific symptoms, it was difficult for our patients to determine the exact timing of the onset of these symptoms, as shown in the index patient of the German cohort [8]. Our study highlights that monitoring of close contacts for early detection of symptom development and immediate isolation of symptomatic patients would also be challenging in real-world settings.

Detailed viral shedding kinetics and infectious doses of SARS-CoV-2 remain to be determined. In our patient cohort, we could not determine the virus titers of the two infectors on the day of virus transmission. The cyclic threshold (Ct) values of the nasopharyngeal swab samples taken on the 4 th day of illness were 18.7 and 18.3 in the index patient and patient 1, respectively. Our study demonstrates that patients with COVID-19 may shed enough virus to transmit from as early as the 1st day of illness. Our data also suggest that the shedding of SARS-CoV-2 from the upper respiratory tract may peak during the prodromal phase of the disease, and the virus shedding kinetics may differ markedly from the severe acute respiratory syndrome coronavirus (SARS-CoV), which show an inverted $\mathrm{V}$ shape, achieving its peak around the tenth day of illness [9].

It is also interesting to note that the first chest radiograph of the index patient and patient 1 failed to show any infiltrates, suggesting the absence of pneumonia at the timing of viral transmission. However, the possibility of pneumonia cannot be fully excluded as a chest radiograph may not be able to detect subtle infiltrates in the lungs due to viral pneumonia [10].

In summary, our patient cohort suggests that (1) patients with COVID-19 may transmit SARS-CoV-2 from the first day of illness, (2) symptoms of infection are similar to the common cold, (3) the transmission may occur in the community during everyday life activities, 
and therefore (4) early detection and isolation of patients with COVID-19 may be challenging.

\section{KEY MESSAGE}

1. Patients with coronavirus disease 2019 transmitted severe acute respiratory syndrome coronavirus 2 on the first day of their illness.

2. The symptoms of infectors were so mild that they tended not to seek medical advice.

3. Transmission occurred in the community during everyday life activities.

\section{Conflict of interest}

No potential conflict of interest relevant to this article was reported.

\section{Acknowledgments}

The authors thank the patients for providing their consent for publication and the public health workers involved in contact tracing and quarantine measures.

\section{REFERENCES}

1. Zhu N, Zhang D, Wang W, et al. A novel coronavirus from patients with pneumonia in China, 2019. N Engl J Med 2020;382:727-733.

2. Wu Z, McGoogan JM. Characteristics of and important lessons from the coronavirus disease 2019 (COVID-19) outbreak in China: summary of a report of 72314 cases from the Chinese Center for Disease Control and Prevention. JAMA 2020 Feb 24 [Epub]. https://doi.org/10.1001/ jama.2020.2648.

3. World Health Organization. Coronavirus disease (COVID
-2019) situation report-69 [Internet]. Geneva (CH): WHO, c2020 [cited 2020 May 21]. Available from: https://www.who. int/emergencies/diseases/novel-coronavirus-2019/situation-reports/.

4. Li Q, Guan X, Wu P, et al. Early transmission dynamics in Wuhan, China, of novel coronavirus-infected pneumonia. N Engl J Med 2020;382:1199-1207.

5. Lim J, Jeon S, Shin HY, et al. Case of the index patient who caused tertiary transmission of COVID-19 infection in Korea: the application of lopinavir/ritonavir for the treatment of COVID-19 infected pneumonia monitored by quantitative RT-PCR. J Korean Med Sci 2020;35:e79.

6. World Health Organization. Coronavirus disease (COVID-19) technical guidance: early investigations protocol [Internet]. Geneva (CH): WHO, c2O20 [cited 2020 May 21]. Available from: https://www.who.int/emergencies/diseases/novel-coronavirus-2019/technical-guidance/early-investigations.

7. Wu P, Hao X, Lau EHY, et al. Real-time tentative assessment of the epidemiological characteristics of novel coronavirus infections in Wuhan, China, as at 22 January 2020. Euro Surveill 2020;25:2000044.

8. Kupferschmidt K. Study claiming new coronavirus can be transmitted by people without symptoms was flawed [Internet]. Washington (DC): American Association for the Advancement of Science, c2020 [cited 2020 May 21]. Available from: https://www.sciencemag.org/news/2020/02/ paper-non-symptomatic-patient-transmitting-coronavirus-wrong.

9. Peiris JS, Chu CM, Cheng VC, et al. Clinical progression and viral load in a community outbreak of coronavirus-associated SARS pneumonia: a prospective study. Lancet 2003;361:1767-1772.

10. Kim JY, Choe PG, Oh Y, et al. The first case of 2019 novel coronavirus pneumonia imported into Korea from Wuhan, China: implication for infection prevention and control measures. J Korean Med Sci 2020;35:e61. 